\title{
EFECTOS DE LA INVERSIÓN EXTRANJERA Y COMPETITIVIDAD EN EL COMERCIO Y PRODUCTIVIDAD DE PAÍSES LATINOAMERICANOS
}

\author{
EFFECTS OF FOREIGN INVESTMENT AND COMPETITIVENESS IN TRADE AND \\ PRODUCTIVITY OF LATIN AMERICAN COUNTRIES
}

\section{EFEITOS DO INVESTIMENTO ESTRANGEIRO E DA COMPETITIVIDADE NO COMÉRCIO E NA PRODUTIVIDADE DOS PAÍSES DA AMÉRICA LATINA}

\section{Bárbara Valenzuela-Klagges ${ }^{1}$ \\ Darcy Fuenzalida-O'Shee ${ }^{2}$}

\begin{abstract}
Resumen
El objetivo del estudio es determinar el efecto de la Inversión Extranjera Directa (IED) y competitividad sobre el comercio bilateral de los países latinoamericanos (Argentina, Brasil, Chile, Colombia, Ecuador, México y Perú) con sus principales destinos comerciales para el período 2006-2012 $\mathrm{y}$, en una segunda etapa, cómo el comercio y la IED afectan en la productividad latinoamericana. Debido a que el flujo comercial de un país con el resto del mundo y su competitividad son variables endógenas, se aplicará un modelo gravitacional ampliado en dos etapas y estimado mediante datos de panel estáticos. La investigación permite concluir que, a mayor IED y logro competitivo global de un país latinoamericano, mayor será su comercio bilateral. Y, en una segunda etapa, se confirma que la apertura comercial influye directamente en la productividad de estos países que, unida a la IED, garantiza un aumento sostenido de su productividad.
\end{abstract}

Doi: https://doi.org/10.15359/eys.25-57.6

Recibido: 21-02-2020. Reenvíos: 28-02-2020, 04-03-2020, 21-04-2020, 24-04-2020. Aceptado: 27-04-2020. Publicado: 11-05-2020.

${ }^{1}$ Académica, Unidad de Postgrado en Negocio, Universidad Mayor, Chile. Doctora en Economía. Correo electrónico: barbara.valenzuela@umayor.cl ORCID https://orcid.org/0000-0002-7584-8183

${ }^{2}$ Académico, Departamento de Ingeniería Comercial, Universidad Técnica Federico Santa María, Chile. Doctor en Administración. Correo electrónico: darcy.fuenzalida@usm.cl ORCID https://orcid.org/0000-0001-5536-4758

Bárbara Valenzuela-Klagges y Darcy Fuenzalida-O'Shee

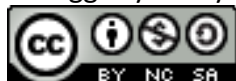

Revista Economía y Sociedad by Universidad Nacional is licensed under a CreativeCommons Reconocimiento-NoComercial- 
Palabras claves: comercio; competitividad; IED; Latinoamérica; modelo gravitacional; productividad

\begin{abstract}
The objective of the study is to determine the effect of Foreign Direct Investment (FDI) and competitiveness on bilateral trade of Latin American countries (Argentina, Brazil, Chile, Colombia, Ecuador, Mexico, and Peru) with their main commercial destinations for the 2006-2012 period. In the second stage, the article analyzes how trade and FDI affect Latin American productivity. Since the commercial flow of a country with the rest of the world and its competitiveness are endogenous variables, an extended gravitational model will be applied in two stages and estimated using static panel data. In conclusion, the greater FDI and global competitive achievement of a Latin American country, the greater its bilateral trade. In addition, in the second stage, it is confirmed that commercial opening directly influences the productivity of these countries which, together with FDI, guarantees a sustained increase in productivity.
\end{abstract}

Keywords: commerce; competitiveness; FDI; Latin America; gravitational model; productivity

\title{
Resumo
}

O estudo tem como objetivo determinar o efeito do Investimento Estrangeiro Direto (IED) e da competitividade no comércio bilateral de países da América Latina (Argentina, Brasil, Chile, Colômbia, Equador, México e Peru) com seus principais destinos comerciais no período de 2006 -2012 e, em uma segunda fase, como o comércio e o IED afetam a produtividade latino-americana. Como o fluxo comercial de um país com o resto do mundo e sua competitividade são variáveis endógenas, um modelo gravitacional expandido será aplicado em duas etapas e estimado por meio de dados em painel estáticos. A pesquisa permite concluir que, quanto maior forem o IED e a conquista competitiva global de um país latino-americano, maior será o comércio bilateral. E, em uma segunda fase, confirma-se que a abertura comercial influencia diretamente na produtividade desses países e, unida ao IED, garante um aumento sustentado por sua produtividade.

Palavras-chaves: comércio; competitividade; IED; América Latina; modelo gravitacional; produtividade 


\section{Introducción}

Como parte introductoria se definirán los conceptos de Inversión Extranjera Directa (IED) y competitividad global y se expondrán, la evolución de estos indicadores en la realidad latinoamericana y evidencias, con el propósito de mostrar la problemática del estudio. Luego se mostrará el objetivo central y la metodología que sustentará el desarrollo de este trabajo.

Krugman \& Obstfeld (2002) define la IED como flujos internacionales de capital que una empresa internacionaliza, al crear una filial en otro país y transferir recursos y el control. Para De la Garza (2005) la IED se origina por empresas que buscan, fuera de su país, ventajas en sus procesos productivos. En la Figura 1 se expone la IED por países, según ingresos altos e ingresos medios y bajos, de acuerdo son la clasificación realizada por el Banco Mundial. Además, se observan las variaciones del IED durante el período 2002-2018, pues son las naciones con ingresos altos las que presentan mayores alzas en los años 2007 y 2015-2016. La baja porcentual mayor se presenta en el 2019 con la crisis subprime, que afectó directamente a Estados Unidos y, por ende, a las naciones con ingresos altos. En el 2015 y 2016, los países con mayores ingresos logran un destacado $4.21 \%$ y $4.14 \%$, respectivamente. No obstante, en el 2018 el desplome de IED en estos alcanzó el $1.1 \%$, mientras que países con ingreso mediano y bajo se mantienen en un $1.93 \%$. Para Jiménez \& Rendón (2012) la IED afecta, de manera positiva, a las economías receptoras por su impacto en el crecimiento de las industrias, lo que incentiva la innovación y, por ende, afecta la competitividad y el Producto Interno Bruto (PIB) del país receptor, sin descartar los posibles efectos en el flujo exportado.

Según Martínez \& Hernández (2012), la IED debe ser considerada como un elemento fundamental para el crecimiento económico de los países receptores, especialmente en aquellos que han adoptado modelos de desarrollo basados en el comercio internacional. Varios estudios de Latinoamérica y del Caribe evidencian que la IED tiene efectos positivos en las exportaciones del estado receptor (Portillo 2005; Martínez \& Hernández, 2012; Toledo 2016). Portillo (2005) afirma que la IED recibida por países integrantes de la Comunidad Andina (Bolivia; Colombia, Ecuador, Perú y Venezuela), durante el período 1990-2000, afectaron en forma positiva sus exportaciones. Toledo (2016) examina la relación entre tres flujos de fondos externos: las remesas, la IED y las exportaciones de América Central y concluye que estas variables convergen en un mayor crecimiento económico. 


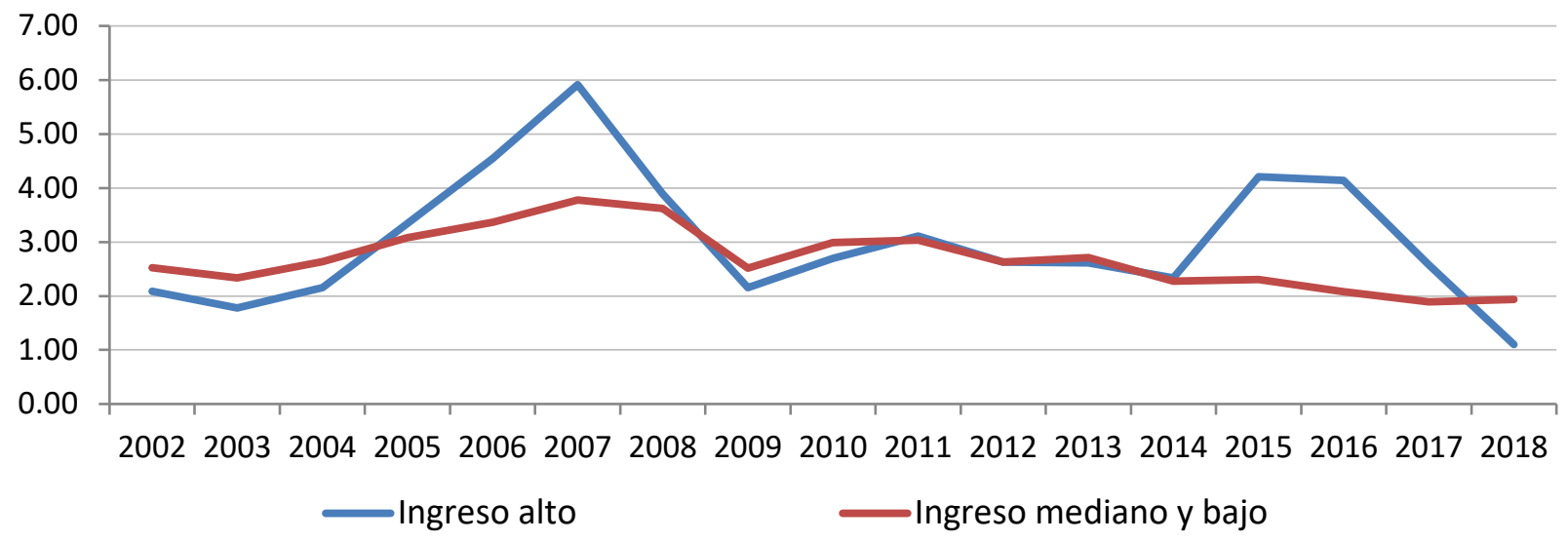

Figura 1. Inversión Extranjera Directa por países según su ingreso, entrada neta de capital (porcentaje del PIB), período 2002-2018. Fuente: Elaboración propia con datos del Banco Mundial.

Por otra parte, el mercado internacional exige competitividad del sector público institucional y del sector privado para atraer IED; fomentar las exportaciones y aumentar la productividad de un país. Varios estudios han validado el efecto de la competitividad global sobre las exportaciones (Yevenes \& Andalaft, 2006; Pérez, 2007; Olivares, 2009; Mayorga \& Martínez, 2008; Overbaugh 2013; Parada, 2011; y Fuenzalida \& Valenzuela, 2016). Por ejemplo, el estudio de Fuenzalida \& Valenzuela (2016) determina el efecto positivo de la calidad institucional de un país sudamericano en el incremento del comercio bilateral y productividad de estos países. Otras investigaciones han validado el efecto de la competitividad sobre la productividad en países Sudamericanos, como los estudios de Kogan (2012) y Fonseca, Muñóz, \& Cleves (2011).

En el Gráfico 2 se observa el porcentaje de exportaciones de productos manufacturados de países con ingreso alto e ingreso mediano-bajo, según la clasificación del Banco Mundial (2020), durante el periodo de 1996 al 2018. Los países con ingresos altos lograron exportar un promedio de $72.4 \%$ entre 1996-2018 en manufactura en relación con el total exportado. Mientras que los países con ingresos medio-bajo, un $52.12 \%$. En promedio durante el periodo 1996-2018 se observa una diferencia mayor al $20 \%$ entre este grupo de países. Esto evidencia la brecha competitiva y estratégica que existe en el mercado mundial. En relación con esta realidad, Mahadevan (2009) analiza el caso de Singapur bajo cinco indicadores: empleo, productividad, producción agregada, exportaciones e importaciones, y concluye que las exportaciones benefician el crecimiento económico de un país en el largo plazo debido al impacto que tienen sobre el PIB y por la adaptación o desarrollo de nuevas tecnologías orientadas al comercio internacional. 


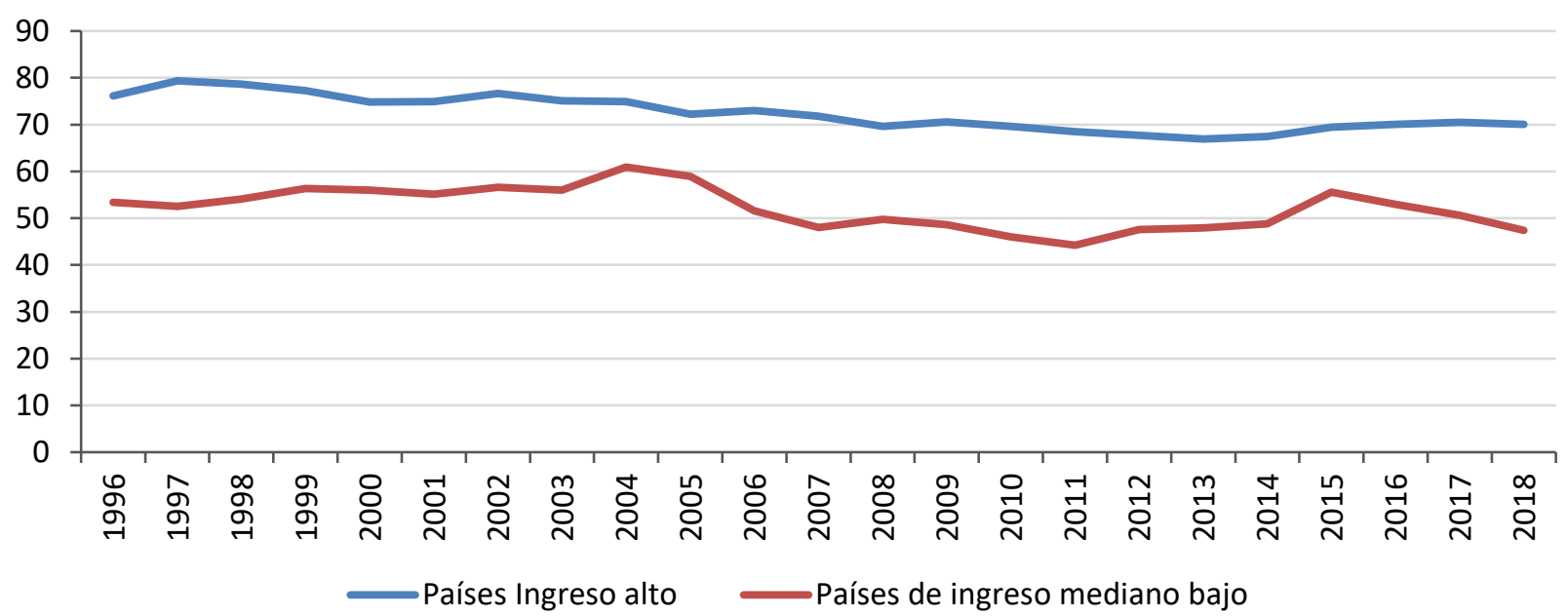

Figura 2. Exportaciones de productos manufacturados de países con ingresos altos e ingresos mediano bajo (\% de las exportaciones de mercancía, 1996-2018). Fuente: Elaboración propia con datos del Banco Mundial.

Dado lo planteado, el objetivo de este estudio es determinar el efecto de la IED y la competitividad sobre el comercio bilateral de los países latinoamericanos (Argentina, Brasil, Chile, Colombia, Ecuador, México y Perú) con sus principales destinos comerciales, para el período 2006-2012 y, en una segunda etapa, cómo el comercio y la IED afectan en la productividad por trabajador latinoamericano. Como el flujo comercial de un país con el resto del mundo y su competitividad son variables endógenas y la IED es exógena, aunque influenciada por variables endógenas, se aplicará una ecuación gravitacional en dos etapas en paneles estáticos. El estudio se sustenta en siete países de Latinoamérica con sus principales 80 socios comerciales, al obtener una muestra de observaciones totales de 7840. Se seleccionaron los siete países de Latinoamérica que presentaban información completa de las variables necesarias para este estudio durante todo el período.

\section{Especificación del modelo}

Para el desarrollo de esta investigación se utilizará una ecuación gravitacional ampliada y adaptada al fenómeno en estudio para el período 2006-2012, con un procedimiento en dos etapas conforme al formulado por Martínez-Zarzoso \& Doyle (2007) y aplicado, por primera vez en Sudamérica, en los estudios de Valenzuela (2011) y Fuenzalida \& Valenzuela (2016). Estos últimos dos estudios, tenían el propósito de medir el impacto de la calidad institucional en el comercio bilateral y productividad de países sudamericanos, en cambio, el principal objetivo de este documento es medir el impacto de la IED y la competitividad global en el comercio bilateral y la productividad por trabajador latinoamericano.

Bárbara Valenzuela-Klagges y Darcy Fuenzalida-O'Shee

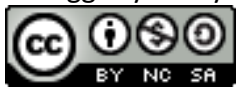

Revista Economía y Sociedad by Universidad Nacional is licensed under a CreativeCommons Reconocimiento-NoComercial- 
Tinbergen (1962) propuso una ecuación de gravedad para explicar los niveles de comercio de un país en términos del tamaño de su economía y la de sus socios comerciales, medidos por Producto Nacional Bruto (PNB) o Producto Interno Bruto (PIB) y distancia geográfica entre ellos medidos en kilómetros. La forma más simple de esta ecuación, que busca determinar el intercambio comercial $\left(E_{i j}\right)$ entre dos países exportador (i) e importador (j), está dada por la Ecuación 1:

$$
E_{i j}=\alpha_{0} Y_{i}^{\left(\alpha_{1}\right)} Y_{j}^{\left(\alpha_{2}\right)} D_{i j}^{\left(\alpha_{3}\right)} \quad \text { Ecuación } 1
$$

Donde, $Y_{i}$ representa el PIB del país exportador, $Y_{j}$ representa el PIB del importador y $D_{i j}$ es la distancia entre ambos países. De acuerdo a la teoría, el PIB es directamente proporcional al intercambio comercial entre países, mientras que la distancia es inversamente proporcional. Los índices $\alpha_{0}, \alpha_{1}, \alpha_{2}$ y $\alpha_{3}$ son parámetros desconocidos. Linnemann (1966) agregó las variables poblacionales del país exportador e importador con el propósito de analizar el desempeño de las economías de escala.

Anderson (1979), Bergstrand (1985) y Helpman \& Krugman (1985) le dieron el sustento teórico a la ecuación gravitacional para modelar fenómenos de flujos comerciales. Otros autores como Mátyás (1997); Cheng \& Wall (1999); Bayoumi \& Eichengreen (1998); Breuss \& Egger (1999), Egger (2000), Marto (2007), suministran especificaciones econométricas de la ecuación gravitacional.

\subsection{Primera etapa}

En la primera etapa y al seguir el método en dos etapas similar al formulado por Martínez-Zarzoso \& Doyle (2007), Valenzuela (2011) y Fuenzalida \& Valenzuela (2016), la Ecuación 2 tiene como variable dependiente el comercio bilateral total de un país exportador con sus principales socios comerciales, en términos relativos a la paridad de poder adquisitivo del Producto Interno Bruto real $^{3}$ del exportador (Trade/PPPGDP) en logaritmos naturales, siendo determinada como:

$$
\begin{gathered}
\ln \left(\frac{\text { TRADEijt }}{\text { PPP GDPit }}\right) \\
=\gamma_{i}+x_{j}+\theta_{t}+\beta_{1} \ln \left(P O P_{i t}\right) \\
+\beta_{2} \ln \left(P O P_{j t}\right)+\beta_{3} \ln \left(A_{i} A_{j}\right)+\beta_{4} \ln \left(\text { Dist }_{i j}\right)+\beta_{5} I d_{i j}+\beta_{6} F_{i j}+\beta_{7} I E D_{i t} \\
+\beta_{8} C G_{i t}+\beta_{9} M E R C O S U R_{i j}+\beta_{10} \text { crisissubprime }+U_{i j} \\
\text { (Ecuación 2) }
\end{gathered}
$$

\footnotetext{
${ }^{3}$ Banco Mundial es la fuente de datos de las variables PIB real, población, área, IED y fuerza productiva. Para el monto exportado entre socios comerciales, se utilizó como fuente la Asociación Latinoamericana de Integración, ALADI, indicadores estadísticos (Comercio bilateral, TRADE).
}

Bárbara Valenzuela-Klagges y Darcy Fuenzalida-O'Shee

Revista Economía y Sociedad by Universidad Nacional is licensed under a CreativeCommons Reconocimiento-NoComercial- 
Donde:

i expresa al país exportador, j al país importador y $t$ el año,

POP : Población de cada país;

A : Área medida en $\mathrm{km}$ al cuadrado

Dist : Distancia entre las capitales del país exportador e importador, medidas en $\mathrm{km}$

IED : Inversión extranjera directa, entrada neta de capitales en millones de dólares

CG : Índice de Competitividad Global publicado por el Foro Económico Mundial

Además, la Ecuación (2) incluye variables ficticias que toman valor de 1 en caso de que apliquen para dicho país o periodo y de 0 en caso de que no lo hagan. Estas variables son las siguientes:

Id : Idioma en común entre país exportador e importador;

$\mathrm{F} \quad$ : Frontera en común entre país exportador e importador;

MERCOSUR : Comercio entre países miembros del bloque regional MERCOSUR

Crisis Subprime: variable tiempo representada por los años 2008-2009

La competitividad del país exportador se representa en el modelo con el Índice de Competitividad Global publicado por el Foro Económico Mundial (2016). Dicho índice analiza doce variables, siendo las siguientes: instituciones; infraestructuras; entorno macroeconómico; salud y educación primaria; educación superior y formación; eficiencia del mercado de bienes; eficiencia del mercado laboral; desarrollo del mercado financiero; preparación tecnológica; tamaño del mercado; sofisticación en materia de negocios e innovación. El índice tiene un intervalo de 0 a 6, siendo 6 la máxima competitividad global. En el Informe del 2016-2017, los tres países más competitivos del mundo fueron Suiza (5.81); Singapur $(5,72)$ y Estados Unidos (5.70). En cuanto a América Latina y el Caribe, los países más competitivos son Chile con 4.64; Perú con 4.23 y México Con 4.41. Venezuela es el país de América Latina y el Caribe peor evaluado (3.27).

Para el análisis se elige el período 2006-2012 debido a dos motivos principales: a) se relaciona con la posibilidad de medir el efecto de la crisis subprime (2008-2009) en países de Latinoamérica y, b) porque los datos de todas las variables incluidas en las ecuaciones 2 y 3 y para todos los países de la muestra, están completos hasta el 2012.

En este estudio optó por dejar una muestra de países cuyo flujo de comercio bilateral no presentaba observaciones durante el período en estudio. Por otra parte, se analizan, mediante matriz de correlación, las variables independientes de la Ecuación 2 que podían presentar entre ellas correlación negativa o positiva alta. Se descarta multicolinealidad. Además, y, siguiendo una de las sugerencias que brindan Santos \& Tenreyro (2006), al no presentar heteroscedasticidad la Ecuación 2, se aplica logaritmo natural para garantizar linealidad.

Bárbara Valenzuela-Klagges y Darcy Fuenzalida-O’Shee

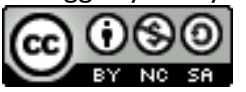

Revista Economía y Sociedad by Universidad Nacional is licensed under a CreativeCommons Reconocimiento-NoComercial- 
Para dejar una muestra de países con flujos positivos, se revisaron los montos de exportaciones e importaciones en detalle; se compararon algunas observaciones cero con informes de las respectivas aduanas, dado que ALADI registra principalmente datos superiores a mil dólares en el flujo comercial anual. Por ello, se excluyeron los países de destino o exportadores que, en un año o más, no presentaban comercio. Por lo que la muestra quedó determinada por 80 países socios comerciales de Argentina, Brasil, Chile, Colombia, Ecuador, México y Perú, abarcando el $89 \%$ del monto total exportado de estos países latinoamericanos durante el período en estudio. Las observaciones anuales son 1120, al incluir el monto exportado e importado por separado. Las observaciones totales son 7840 .

\subsection{Segunda etapa}

En la segunda etapa, es planteada la Ecuación 3 que relaciona productividad por trabajador del país latinoamericano con el proxy del comercio estimado en la ecuación anterior (Ecuación 2), población e IED. La Ecuación 3 es la siguiente:

$$
\ln \left(\frac{P P P G D P_{c t}}{L_{c t}}\right)=\alpha_{0}+\alpha_{1} \ln \left(T R A D E_{c t}\right)+\alpha_{2} \ln \left(P O P_{c t}\right)+\alpha_{3}\left(I E D_{c t}\right)+\mu_{c t}
$$

(Ecuación 3)

Siendo:

PPPGDPC : : Labor productiva por trabajador del país en estudio (c)

LC $\quad$ : Fuerza productiva en el país en estudio (c)

TRADE : Constituye el proxy del comercio que viene dada por la predicción obtenida a partir de la ecuación anterior (Ecuación 2)

POP : Población del país en estudio (c)

IED : Inversión extranjera directa, entrada neta de capitales en millones de dólares

Se estimarán las ecuaciones ( 2 y 3) mediante datos de panel estáticos con efectos fijos y aleatorios, realizando los respectivos test de verificación.

\section{Resultados}

En la Tabla 1 se muestran los resultados de la Ecuación 2, mediante datos de panel efectos fijos y aleatorios, que incluyen solo las variables con significatividad. Las variables no significativas se van descartando según metodología de Donoso, Valenzuela, Soto, Muñoz, \& Valenzuela (2020), desde la que presenta mayor error a la de menor error. Es posible observar que los coeficientes de las variables expuestas en la Tabla 1, presentan signos esperados y la mayoría son estadísticamente significativos. Los resultados con efectos aleatorios y expuestos en la Tabla 1, muestran coeficientes significativos semejantes a los observados en regresión agrupada 
mediante pool no expuesta en la tabla, pero con diferencias en los valores de sus coeficientes. Al aplicar la prueba de Breusch y Pagan el $p$-value (Prob>chi2=0.000) nos confirma que los efectos aleatorios son relevantes y es preferible antes del agrupado pool. Por otra parte, la prueba $\mathrm{F}$ de significatividad de los efectos fijos reporta $\mathrm{p}$-value (Prob $>\mathrm{F}=0.000)$, por lo que se descarta la estimación por medio del agrupado pool. Con el propósito de comparar las diferencias entre el coeficiente de efectos fijos y aleatorios, se aplica el test de Hausman, al obtener un Prob> Chi2=0.058. Por consiguiente, se ratifica que el método de efectos aleatorios es más conveniente que el de efectos fijos. En la prueba de Durbin, Wu y Hausman no se detecta endogeneidad, por lo que no se recomienda utilizar el panel dinámico. El coeficiente de determinación de la estimación de la Ecuación 2 mediante datos de panel con efectos aleatorios es 0.71.

Tabla 1

Resultados estimación Ecuación 2 (Datos de panel con efectos fijos y aleatorios)

\begin{tabular}{lrr}
\hline \multicolumn{1}{c}{ Variables } & Efectos fijos & \multicolumn{1}{c}{ Efecto aleatorio } \\
\hline C & & $-7.2^{* * *}(9.6)$ \\
Población i & $-0.21^{* * *}(9.4)$ & $-0.3^{*}(8.4)$ \\
Población j & $0.6^{* * *}(5.4)$ & $0.4^{* * *}(2.2)$ \\
Área (i)*Área(j) & & $-0.03^{* * *(2.1)}$ \\
Distancia Geográfica & & $-0.2^{* * *}(6.8)$ \\
Idioma en común & & $0.3^{* * *}(3.1)$ \\
Frontera en común & & $0.8^{* * *}(8.8)$ \\
IED & $0.7^{* *}(2.1)$ & $0.81^{* *}(4.2)$ \\
CG & $0.09^{* *}(1.9)$ & $0.11^{* *}(1.9)$ \\
\hline Crisis Subprime & & $-0.03^{*}(1.9)$ \\
\hline R2 & & $\mathbf{0 . 7 1}$ \\
\hline
\end{tabular}

Nota: Se expresan el valor del coeficiente y estándar de error entre paréntesis, respectivamente. La significatividad del coeficiente se expresa con *** (probabilidad de error menor a $0.5 \%$ ); $* *$ (probabilidad de error entre un $0.5 \%$ y $2.5 \%$ ) ${ }^{*}$ (probabilidad $2.5 \%$ a 5 \%). Fuente: Estimación mediante STATA, elaboración propia.

Los coeficientes de las variables: "población del exportador", "áreas" y la" distancia geográfica entre exportador e importador", tienen una relación negativa en el flujo comercial. Mientras que los coeficientes de las variables "población del importador"; "idioma y frontera en común"; "IED y competitividad global", son positivos y significativos, siendo el coeficiente de IED mayor que el resto. El coeficiente de la variable MERCOSUR no presenta significatividad. Los resultados de algunas de ellas presentan valores semejantes a lo observado en el estudio de Fuenzalida \& Valenzuela (2016), como población del exportador, área y competitividad global.

La crisis subprime presenta coeficiente negativo y significativo, con un bajo efecto en el flujo comercial. 
La Ecuación 3 se estima mediante panel con efectos fijos y aleatorios. Al aplicar el test de Hausman se obtiene un Prob $>$ Chi2 $=0.002$, que ratifica el método de efectos fijo. En la Tabla 2 se exponen los resultados de la Ecuación 3 a través de datos de panel con efectos fijos. El coeficiente de determinación es 0.68 .

Tabla 2

Resultado estimación Ecuación 3, datos de panel efectos fijos

\begin{tabular}{lc}
\multicolumn{1}{c}{ Variables } & Efectos fijos \\
\hline TRADE & $0.42^{* * *}(4.6)$ \\
POP & $0.02^{*}(2.2)$ \\
IED & $0.23^{* * *}(4.4)$ \\
\hline R2 & $\mathbf{0 . 6 8}$ \\
\hline
\end{tabular}

Nota: Se expresa el valor del coeficiente y estándar de error entre paréntesis, respectivamente. La alta significatividad del coeficiente se expresa con *** (probabilidad de error menor a $0.5 \%$ ); ${ }^{* *}$ (probabilidad de error entre un $0.5 \%$ y $2.5 \%$ ) y * (probabilidad 2.5 \% a 5 \%). Fuente: Estimación mediante STATA, elaboración propia.

Los resultados de la Ecuación 3 expuestos en la Tabla 2, indican que los coeficientes de las variables apertura comercial (TRADE), población e IED son positivos y significativos. Al igual que lo planteado por los autores Valenzuela (2011) y Fuenzalida \& Valenzuela (2016), la apertura comercial es fundamental para el aumento productivo de los países latinoamericanos.

Adicionalmente, se confirma que la IED no solo afecta el flujo comercial de los países latinoamericanos, sino que, además, afecta en la productividad de los países latinoamericanos. Es destacable que, entre estos dos efectos de la IED, el mayor es al flujo comercial.

\section{Conclusiones}

Este estudio permite concluir que la "IED y la competitividad global" afecta positivamente en el flujo comercial de países latinoamericanos. Estos resultados son conformes a lo planteado por Portillo (2005); Martínez \& Hernández (2012) y Toledo (2016), la IED es fundamental para incrementar el flujo de comercio internacional del país $y$, de forma directa e indirecta, la labor productividad por trabajador, principalmente en países en que se sustentan en ese comercio. En cuanto a lo observado en la competitividad de países latinoamericanos, los resultados confirman el efecto positivo en la apertura comercial de un país, siendo coherente con lo planteado los

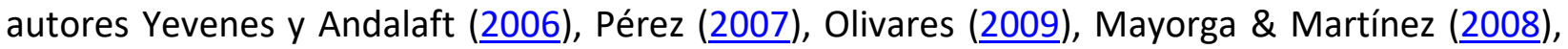
Overbaugh (2013), Parada (2011), Fuenzalida \& Valenzuela (2016) y Cuadros (2018). Para O'Connor (2008) la inserción exportadora de Argentina está directamente relacionada con la competitividad, al exponer que en los rubros donde el país es competitivo, el grado de inserción es mayor. 
Para mejorar la competitividad global de un país latinoamericano se sugiere enfocar las políticas públicas en la calidad de sus instituciones, lo que garantiza transparencia y eficiencia de los recursos públicos; optimizar la infraestructura pública para facilitar el comercio, como puertos, la conductividad vial y líneas férreas; lograr mantener un entorno macroeconómico estable; cumplir con la cobertura en la salud y la educación primaria y superior, que avale un capital humano profesionalizado y con bienestar; garantizar la propiedad privada y facilitar la creación de nuevos giros comerciales. O'Connor (2008) plantea otras estrategias para fomentar la competitividad de Argentina que puede replicarse en otros países latinoamericanos, como desarrollar las cadenas de valor agroindustriales e industriales; aplicar políticas para ampliar la oferta productiva que resuelva el problema de la exportación de bienes-salario y controlar el mercado informal.

Visiblemente, la competitividad global por sectores productivos llevará a mejorar la intensidad competitiva por muchas identidades privadas, las cuales estimularán a otros participantes del mercado que copiarán dichas estrategias competitivas, que potenciarán el comercio interno como el externo.

La crisis subprime tiene un efecto negativo en el flujo comercial de la región en estudio y, por ende, en la productividad por trabajador. Esto permite proyectar que la crisis sanitaria mundial, de 2020, afectará, externa e internamente, y de forma negativa el flujo comercial de los países en estudio. Esto conllevará a menor labor productiva por trabajador.

El efecto de países fronterizos para el flujo comercial entre países de Latinoamérica sigue siendo un factor fundamental. Esto puede deberse al bajo costo de traslado internacional, mayor comunicación comercial entre países y acuerdos de complementación económica o asociación que facilitan el intercambio. Adicionalmente, se observa que el idioma en común entre socios comerciales logra afectar positivamente en su flujo comercial, aunque en menor medida que el efecto frontero en común. En este sentido, es imperativo aumentar las posibilidades de accesibilidad al estudio de una lengua extranjera, que permita mayor comunicación con núcleos industriales extranjeros o de mayor concentración poblacional y, en consecuencia, aumentar el flujo comercial internacional.

Adicionalmente, los resultados permiten concluir que la apertura comercial e IED tienen un efecto positivo en la labor productiva por trabajador de la región, siendo la apertura comercial la de mayor impacto. Estos resultados son coherentes con trabajos que han interactuado la liberalización del comercio con la productividad, como los de Yeaple (2005) y Melitz \& Octaviano (2008).

Este resultado permite concluir que, si un país latinoamericano quiere aumentar la labor productiva por trabajador, se sugiere enfocar esfuerzos en mejorar los factores que intervienen 
positivamente en el flujo comercial con sus principales socios comerciales $y$, en segundo orden, atraer IED. Aunque existen riesgos microeconómicos que deben tenerse en cuenta.

El peligro de liberar el comercio es que las empresas de baja productividad tienen más posibilidades de fracasar y las de alta productividad tienen una probabilidad mucho mayor de sobrevivir y crecer. Por ello, es fundamental acompañar la liberalización del comercio con políticas que fomenten la competitividad global del país.

Este estudio motiva a continuar analizando el comportamiento de la IED y la competitividad por sectores productivos y los efectos de las empresas transnacionales, que permita con detalle verificar el impacto por sector productivo y posibles medidas comerciales.

\section{Referencias}

Anderson, J. (1979). A Theoretical Foundation for the Gravity Equation. The American Economic Review, 69(1), 106-116. Recuperado de https://www.jstor.org/stable/pdf/1802501.pdf

Banco Mundial. (2020). Datos de libre acceso del Banco Mundial. Recuperado de https://datos.bancomundial.org/

Bayoumi, T., \& Eichengreen, B. (1998). Exchange rate volatility and intervention: implications of the theory of optimum currency areas. Journal of International Economics, 45(2), 191202. Recuperado de https://www.sciencedirect.com/science/article/abs/pii/S0022199698000324

Bergstrand, J. (1985). The Gravity equation in international Trade: Some Microeconomic Foundations and Empirical Evidence. The Review of Economics and Statistics, 67(3), 474-481. Doi: https://doi.org/10.2307/1925976

Breuss, F., \& Egger, P. (1999). How Reliable Are Estimations of East-West Trade Potentials Based on Cross-Section Gravity Analyses? Empírica, 26, 81-94. Doi: https://doi.org/10.1023/A:1007011329676

Cheng, I., \& Wall, H. (1999). Controlling for Heterogeneity in Gravity Models of Trade. Doi: https://doi.org/10.2139/ssrn.234349

Cuadros, I. (2018). La eficiencia del mercado de bienes en la competitividad global y las exportaciones de uva fresca del 2011 al 2017. En Repositorio de la Universidad César Vallejo. Recuperado de http://repositorio.ucv.edu.pe/handle/UCV/36470 
De la Garza, U. (2005). La Inversión Extranjera Directa (IED), teorías y prácticas. Innovaciones de Negocios, 2(3), 17-33. Recuperado de http://revistainnovaciones.uanl.mx/index.php/revin/article/view/23

Donoso Ovalle, R., Valenzuela Klagges, B., Soto Bubert, A., Muñoz Pavlov, V., \& Valenzuela Klagges, I. (2020). Producción chilena de Molibdeno: Influencia en el mercado mundial y su comportamiento exportador (2007-2016). Revista de Economía del Rosario, 23(1), 149-172.

Doi: https://doi.org/10.12804/revistas.urosario.edu.co/economia/a.8629

Egger, P. (2000). A note on the Proper Econometric Specification of Gravity Equation. Economics Letters, 66(1), 25 -31. Doi: https://doi.org/10.1016/S0165-1765(99)00183-4

Fonseca, J., Muñóz, N., \& Cleves, J. (2011). El sistema de gestión de calidad: elemento para la competitividad y la sostenibilidad de la producción agropecuaria colombiana. Revista de Investigación Agraria y Ambiental, 2(1), 9-22. Doi: https://doi.org/10.22490/21456453.909

Fuenzalida, D., \& Valenzuela, B. (2016). Calidad institucional y competitividad en el comercio y producción de países sudamericanos. Perfiles Económicos, (2), 29-51. Doi: https://doi.org/10.22370/rpe.2016.2.1204

Helpman, E., \& Krugman, P. (1985). Market Structure and Foreign Trade. Increasing Returns, Imperfect Competition, and the International Economy. (Primera ed.). Cambridge: The MIT Press. Recuperado de https://mitpress.mit.edu/books/market-structure-andforeign-trade

Jiménez, D., \& Rendón, H. (2012). Determinantes y efectos de la inversión extranjera directa: revisión de literatura. Ensayos de economía, 22(41), 109-128. Recuperado de https://revistas.unal.edu.co/index.php/ede/article/view/35873/43643

Kogan, J. H. (2012). Infraestructura, integración física y competitividad internacional de la Región Sudamericana. En Santa, R. (Ed.). Integración física sudamericana diez años después: impacto e implementación en el Perú. (Primera ed., 73-88). Lima: Universidad del Pacífico. Recuperado de http://repositorio.up.edu.pe/handle/11354/1010 
Krugman, P., \& Obstfeld, M. (2002). International Factor Movements. En Krugman, P., \& Obstfeld, M. (Eds.). International Economics: Theory and policy. (Sexta ed., 181-183). Estados Unidos: Addison Wesley. Recuperado de https://www.ssc.wisc.edu/ munia/464/Intertemporal\%20Trade.pdf

Linnemann, H. (1966). An Econometric Study of International Trade Flows. (Primera ed.) Amsterdam: North Holland publishing Co. Recuperado de https://books.google.com.uy/books/about/An_Econometric_study_of_international _tr.html?id=nCi3AAAAIAAJ

Mahadevan, R. (2009). The Sustainability of Export-Led Growth: The Singaporean Experience. The Journal of Developing Areas, 43(1), 233-247. Doi: https://doi.org/10.1353/jda.0.0034

Martínez-Zarzoso, I., \& Doyle, E. (2007). Trade, Productivity and Institutional Quality: Issue and Empirics. German Development Economics Conference, Göttingen 2007 from Verein für Socialpolitik. Research Committee Development Economics. Recuperado de https://www.econstor.eu/handle/10419/19876

Martínez, J., \& Hernández, R. (2012). La inversión extranjera directa en Costa Rica: Factores determinantes y efectos en el desarrollo nacional y regional. En Repositorio institucional CONARE. Recuperado de http://repositorio.conare.ac.cr/handle/20.500.12337/906

Marto, A. (2007). Empirical Examination of the Gravity Model in two Different Contexts: Estimation and Explanation. Jahrbuch für Regionalwissenschaft, 27, 103-127. Recuperado de https://link.springer.com/article/10.1007\%2Fs10037-007-0013-8

Mayorga, J., \& Martínez, C. (2008). Paul Krugman y el nuevo comercio internacional. Criterio Libre, 8, 73-86. Recuperado de https://dialnet.unirioja.es/servlet/articulo?codigo=4547087

Melitz, M., \& Ottaviano, G. (2008). Market Size, Trade, and Productivity. The Review of Economic Studies, 75(1), 295-316. Doi: https://doi.org/10.1111/j.1467-937X.2007.00463.x

Olivares, O. (2009). Competitividad y calidad factores críticos que contribuyen al éxito de empresas de menor tamaño en mercados globales de país emergente. Revista Chilena de Economía y Sociedad, 3(1), 29-51. Recuperado de https://rches.utem.cl/articulos/competitividad-y-calidad-factores-criticos-quecontribuyen-al-exito-de-empresas-de-menor-taman\%CC\%83o-en-mercadosglobales-de-pais-emergente/ 
O'Connor, E. (2008). Problemas de competitividad de las exportaciones argentinas. Ensayos de Política Económica, 1(2), 106-125. Recuperado de http://erevistas.uca.edu.ar/index.php/ENSAYOS/article/view/2452

Overbaugh, S. (2013). National culture, country-level competitiveness, and economic development. International Journal of Business \& Economics Perspectives, 8(1), 93108. https://go.gale.com/ps/anonymous?id=GALE\%7CA335974243\&sid=googleScholar\&v $=2.1 \& i t=r \&$ linkaccess $=a b s \& i s s n=1931907 X \& p=A O N E \& s w=w$

Parada, Á. (2011). El comercio internacional un instrumento para alcanzar el desarrollo de Costa Rica. Economía y Sociedad,16(39-40), 109-132. Recuperado de https://www.revistas.una.ac.cr/index.php/economia/article/view/4903

Pérez, R. (2007). Competitividad empresarial de cara a la exportación. Una conceptualización necesaria. Economia y Desarrollo, 142(2), 67-79. Recuperado de https://www.redalyc.org/articulo.oa?id=425541311003

Portillo, S. (2005). Efecto de las inversiones extranjeras directa de España sobre el comercio de la comunidad Andina de naciones. Estudios Económicos de Desarrollo Internacional, 5(2), 49-62. Recuperado de https://www.redalyc.org/pdf/165/16550203.pdf

Santos, J., \& Tenreyro, S. (2006). The log of gravity. Review of Economics and Statistics, 88(4), 641658. Doi: https://doi.org/10.1162/rest.88.4.641

Tinbergen, J. (1962). Shaping the World Economy: suggestions for an International Economic Policy. (Primera ed.). New York: Twentieth Cetury Fund. Recuperado de https://repub.eur.nl/pub/16826

Toledo, W. (2016). Los determinantes de las exportaciones, la inversión extranjera directa y las remesas en ocho economías Latinoamericanas. Atlantic Review of Economics, 1(1), 124. Recuperado de https://dialnet.unirioja.es/servlet/articulo?codigo $=5569036$

Valenzuela, B. (2011). Comercio y calidad institucional en el crecimiento económico del cono sur americano: cuestiones y desafíos. Revista Pilquen. Sección Ciencias Sociales, 14(1), 1$8 . \quad$ Recuperado de http://revele.uncoma.edu.ar/htdoc/revele/index.php/Sociales/article/view/1583 
WorlD Economic Forum (2016) The Global Competitiveness Report. Recuperado de http://www3.weforum.org/docs/GCR2016-

2017/05FullReport/TheGlobalCompetitivenessReport2016-2017 FINAL.pdf

Yeaple, S. (2005). A simple model of firm heterogeneity, international trade, and wages. Journal of International Economics, 65(1), 1-20. Doi: https://doi.org/10.1016/j.jinteco.2004.01.001

Yevenes Subiabre, A., \& Andalaft Chacur, A. (2006). Competitividad territorial y estrategia empresarial: el caso de la industria forestal exportadora de la región del Bío Bío. Horizontes Empresariales, 5(1), 39-51. Recuperado de http://revistas.ubiobio.cl/index.php/HHEE/article/view/2063 